\title{
Differentiating tuberculosis from sarcoidosis by sonographic characteristics of lymph nodes on endobronchial ultrasonography: A study of 165 patients
}

\author{
Sahajal Dhooria, MD, DM, ${ }^{\mathrm{a}}$ Ritesh Agarwal, MD, DM, ${ }^{\mathrm{a}}$ Ashutosh N. Aggarwal, MD, DM, ${ }^{\mathrm{a}}$ \\ Amanjit Bal, MD, ${ }^{\mathrm{c}}$ Nalini Gupta, MD, ${ }^{\mathrm{b}}$ and Dheeraj Gupta, MD, DM ${ }^{\mathrm{a}}$
}

\begin{abstract}
Objective: The differential diagnosis of sarcoidosis and tuberculosis is difficult, especially in countries with a high tuberculosis burden. We hypothesized that sonographic features on endobronchial ultrasonography (EBUS) would help in differentiating tuberculosis from sarcoidosis. In this study, the endosonographic features of tuberculosis and sarcoidosis are compared.
\end{abstract}

\begin{abstract}
Methods: This was a retrospective analysis of prospectively collected data of patients with intrathoracic lymphadenopathy who underwent EBUS-guided transbronchial needle aspiration (TBNA), and were finally diagnosed with sarcoidosis or tuberculosis. Sonographic features such as size, shape (round or oval), margin (distinct or indistinct), echogenicity (heterogeneous or homogeneous), presence or absence of a central hilar structure, and coagulation necrosis sign were recorded and compared in the 2 groups.
\end{abstract}

\begin{abstract}
Results: During the study period, 249 EBUS-guided TBNA procedures were performed and a diagnosis of sarcoidosis $(\mathrm{n}=118)$ or tuberculosis $(\mathrm{n}=47)$ was made in 165 patients. A total of 358 lymph node stations were examined. Heterogeneous echotexture $(53.4 \%$ vs $12.6 \%, P<.001)$ and coagulation necrosis $(26.1 \%$ vs $3.3 \% ; P<.001)$ were significantly higher in tuberculous lymph nodes. A combination of a positive tuberculin skin test (TST) and either heterogeneous echotexture or coagulation necrosis sign had specificity of $98 \%$ and positive predictive value of $91 \%$ for a diagnosis of tuberculosis.
\end{abstract}

Conclusions: Sonographic features of heterogeneous echotexture or coagulation necrosis in the lymph nodes on EBUS are fairly specific for tuberculosis. Along with a positive TST, these features strongly favor a diagnosis of tuberculosis over sarcoidosis. (J Thorac Cardiovasc Surg 2014;148:662-7)

Sarcoidosis and tuberculosis are 2 granulomatous disorders that closely resemble each other. ${ }^{1}$ The presence of intrathoracic lymphadenopathy with or without lung infiltrates is a feature shared by these 2 conditions. ${ }^{2,3}$ Differentiation between these 2 granulomatous disorders is mainly dependent on subtle differences in clinical presentation, microbiological investigations for Mycobacterium tuberculosis, the presence or absence of hypersensitivity to purified protein derivative (PPD), and the microscopic features of the granulomas identified on cytologic or histologic specimens. ${ }^{4}$ Blind transbronchial needle aspiration (TBNA) has been used traditionally to obtain tissue specimens from intrathoracic lymph nodes in patients with suspected sarcoidosis and tuberculosis. $^{5-7}$ With the advent of endobronchial ultrasonography (EBUS), the yield of TBNA from mediastinal/hilar lymph

From the Departments of Pulmonary Medicine, ${ }^{\mathrm{a}}$ Cytology, ${ }^{\mathrm{b}}$ and Histopathology, Postgraduate Institute of Medical Education and Research, Chandigarh, India.

Disclosures: Authors have nothing to disclose with regard to commercial support.

Received for publication Aug 21, 2013; revisions received Jan 7, 2014; accepted for publication Jan 21, 2014; available ahead of print Feb 16, 2014.

Address for reprints: Dheeraj Gupta, MD, DM, Department of Pulmonary Medicine, Postgraduate Institute of Medical Education and Research, Chandigarh-160012, India (E-mail: dheeraj1910@gmail.com).

0022-5223/\$36.00

Copyright (c) 2014 by The American Association for Thoracic Surgery

http://dx.doi.org/10.1016/j.jtcvs.2014.01.028 nodes has significantly improved. ${ }^{8,9}$ In a recent metaanalysis, we found EBUS TBNA to be a safe and efficacious modality in the diagnosis of sarcoidosis. ${ }^{10}$

The sonographic features of intrathoracic lymph nodes on EBUS have been found to be useful in differentiating benign from malignant involvement. ${ }^{11,12}$ However, the role of sonographic appearances in the differential diagnosis of 2 common benign causes of mediastinal lymphadenopathy (sarcoidosis and tuberculosis) has not been investigated. Intrathoracic lymph nodes secondary to tuberculosis demonstrate low-density centers on computed tomography (CT) of the chest. ${ }^{13}$ We hypothesized that EBUS characteristics of mediastinal lymph nodes could help in differentiating between sarcoidosis and tuberculosis. In this study, the endosonographic features of tuberculosis and sarcoidosis are compared and their usefulness in the differential diagnosis of the 2 clinical mimics is analyzed.

\section{METHODS \\ Patients}

This was a retrospective analysis of prospectively collected data of patients undergoing EBUS TBNA between October 1, 2011, and June 1, 2013. The study protocol was approved by the Ethics Review Committee and written informed consent was obtained from all patients. Consecutive patients presenting to the Bronchoscopy Suite of this Institute who had intrathoracic lymph node enlargement on CT of the chest and were 

Abbreviations and Acronyms
$\mathrm{CT}=$ computed tomography
$\mathrm{EBB}=$ endobronchial biopsy
EBUS $=$ endobronchial ultrasonography
NAA $=$ nucleic acid amplification
$\mathrm{OR}=$ odds ratio
PPD = purified protein derivative
$\mathrm{SD}=$ Standard deviation
TBLB $=$ transbronchial lung biopsy
TBNA $=$ transbronchial needle aspiration
TST $=$ tuberculin skin test

with standard biopsy forceps (FB19C, Olympus, Japan) were used to perform the biopsies.

\section{EBUS Image Features of Lymph Nodes}

The endobronchial ultrasonographic image classification system proposed by Fujiwara and colleagues was used to define the lymph node characteristics. ${ }^{11}$ The following nodal features were recorded: (1) size (in millimeters) of the longest dimension (long-axis size) and a dimension perpendicular to it (short-axis size); (2) shape on visual inspection (oval or round); (3) margin (distinct or indistinct): when most of the margin $(>50 \%)$ was clearly visualized, the lymph node was classified as distinct; (4) echogenicity (homogeneous or heterogeneous): a node was labeled as heterogeneous if there were multiple small areas of varying echogenicity; (5) presence or absence of a central hilar structure, which is a linear avascular hyperechoic area in the center of a lymph node; and (6) the presence or absence of coagulation necrosis defined as the presence of 1 or more large hypoechoic areas within a lymph node with absence of blood flow on Doppler (Figure 1). ${ }^{16}$ The EBUS characteristics of the lymph nodes were determined and recorded after being agreed on by at least 2 of the operators.

列 the following were excluded: pregnancy, hypoxemia (pulse oximetric saturation $<90 \mathrm{~mm} \mathrm{Hg}$ in room air), a deranged coagulation profile, or failure to provide informed consent. All patients underwent a detailed clinical evaluation, laboratory tests (complete blood count, coagulation profile, liver and renal function tests, and angiotensin-converting enzyme levels), chest radiography, and CT of the chest. Tuberculin skin testing (TST) with 5 tuberculin units of PPD was performed in all patients and a value of $10 \mathrm{~mm}$ or higher was considered positive.

\section{EBUS TBNA Procedure}

EBUS procedures were performed by consultants experienced in the EBUS technique or by pulmonary fellows under direct supervision of the consultants. Lymph node stations were classified according to the International Association for the Study of Lung Cancer map, both on CT and EBUS. ${ }^{14}$ The procedure was performed on an outpatient basis under conscious sedation and analgesia (intravenous midazolam and pentazocine in doses sufficient to maintain sedation and cough control). Patients were administered $0.6 \mathrm{mg}$ atropine and $25 \mathrm{mg}$ promethazine intramuscularly followed by nebulized lignocaine ( $4 \%$ solution) immediately before the procedure. Topical $10 \%$ lignocaine was sprayed over the oropharynx augmented with $2 \%$ lignocaine solution instilled over the vocal cords and the airways. Monitoring of pulse rate, respiratory rate, pulse oximetric saturation, and blood pressure was performed throughout the procedure. The convex probe EBUS scope (BF-UC 180F, Olympus Medical Systems, Japan) with a $7.5 \mathrm{MHz}$ convex transducer and a compatible endoscopic ultrasound scanner (EU-ME1, Olympus Medical Systems, Japan) were used.

EBUS TBNA was performed in a standard fashion in the supine position by the oral route using a dedicated, disposable, 21-gauge, Vizishot needle (NA-201SX-4021, Olympus Medical Systems, Japan) under real-time sonographic and endoscopic visualization. ${ }^{15}$ The aspirate was then blown on to a glass slide by pushing air using a $20-\mathrm{mL}$ syringe. Aspirated material was also obtained for cell block and mycobacterial cultures (in normal saline using a mycobacterial growth indicator tube [MGIT]). If a histologic core was obtained, it was immediately fixed in $10 \%$ formalin. A maximum of 3 aspirates was obtained from each lymph node. In patients with multiple enlarged lymph nodes, the largest lymph nodes were sequentially accessed. On-site cytologic assessment for adequacy of the aspirate was unavailable.

The decision to perform an endobronchial biopsy (EBB) and transbronchial lung biopsy (TBLB) was left to the discretion of the bronchoscopist. In general, EBB was obtained if there were endobronchial abnormalities or in patients with clinical suspicion of sarcoidosis. A TBLB was obtained if there were any parenchymal abnormalities on the CT scan or in those with a clinical diagnosis of sarcoidosis. A conventional fiber optic bronchoscope (BF-1T20 or BF-TE2, Olympus, Japan; FB-19TV, Pentax, Japan) along

\section{Diagnosis of Sarcoidosis and Tuberculosis}

A final diagnosis of sarcoidosis was made on the presence of all of the following criteria: (1) consistent clinical and radiological presentation; (2) demonstration of nonnecrotizing granulomas on either EBUS TBNA, TBLB, or EBB along with negative acid-fast bacilli and fungal stains; and no growth of mycobacteria on MGIT; (3) clinical and radiological response after treatment with glucocorticoids. ${ }^{17}$ A diagnosis (1) necrotizing granulomatous inflammation or the presence of acid-fast bacilli on microscopy or a positive culture for M tuberculosis; (2) clinicoradiological response to antituberculosis treatment.

\section{Statistical Analysis}

Statistical analysis was performed using the commercial statistical package StatsDirect (version 2.7.2, StatsDirect Ltd, Altrincham, United Kingdom, 2005. http://www.statsdirect.com). Data were expressed as the mean \pm standard deviation (SD) or number with percentage. Differences between continuous variables in the 2 groups were compared using the Mann-Whitney $U$ test; differences between categorical data were compared using the $\chi^{2}$ test or the Fisher exact test. The performance characteristics of various endosonographic signs and the TST are presented as sensitivity, specificity, and predictive values. Agreement for the sonographic characteristics between lymph node stations in the same patient was evaluated using the kappa statistic. Multivariate logistic regression analysis was performed to define factors predicting the diagnosis of tuberculosis.

\section{RESULTS}

A total of 249 EBUS TBNA procedures were performed in 245 patients. Four patients underwent the procedure twice as the first procedure was nondiagnostic. These 4 patients (amounting to 8 procedures) were not considered for analysis. The mean (SD) age of the remaining 241 patients (107 [44.4\%] women) was 44.7 (13.9) years. Of the 241 patients, a diagnosis of malignancy was made in 26 ; in 50 patients, no definite diagnosis was made after EBUS and 6 months of follow-up. A final diagnosis of sarcoidosis or tuberculosis was made in 165 patients (118 sarcoidosis and 47 tuberculosis) from the results of pathologic examination and microbiology. TBLB was performed in 110 of the 165 patients and EBB was performed of tuberculosis was based on demonstration of all of the following: 


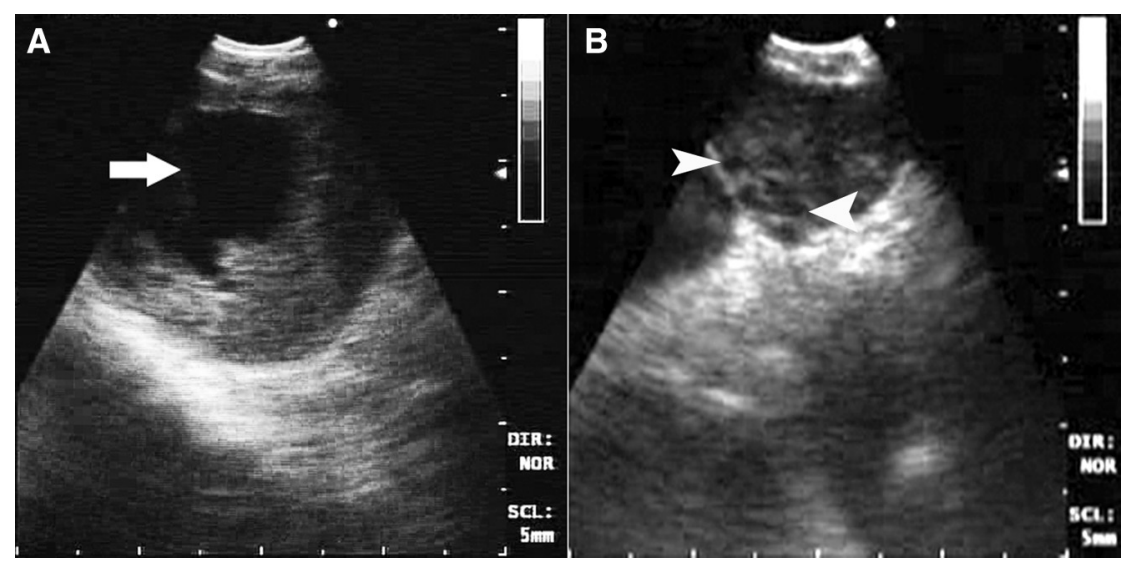

FIGURE 1. Coagulation necrosis sign (A, arrow) and heterogeneous lymph node echotexture (B, arrowheads) in 2 different patients with a diagnosis of tuberculosis. Frank pus was aspirated in both patients and cultures grew $M$ tuberculosis.

in 108 patients. In patients with tuberculosis, necrotizing granulomas were demonstrated in 33 patients, cultures were positive for $M$ tuberculosis in 12 patients, and acid-fast bacilli were demonstrated in 9 patients. The baseline characteristics of these 165 patients are shown in Table 1. Patients with tuberculosis were significantly younger than patients with sarcoidosis and had a higher prevalence of smoking. A positive TST (induration more than $10 \mathrm{~mm}$ ) was seen in a larger proportion of patients with tuberculosis.

TABLE 1. Baseline characteristics of the patients and number of lymph nodes sampled at various stations

\begin{tabular}{|c|c|c|c|c|}
\hline & $\begin{array}{c}\text { Sarcoidosis } \\
(\mathbf{n}=\mathbf{1 1 8})\end{array}$ & $\begin{array}{l}\text { Tuberculosis } \\
(\mathbf{n}=47)\end{array}$ & $\begin{array}{c}\text { Total } \\
(\mathrm{n}=165)\end{array}$ & $\begin{array}{c}P \\
\text { value }\end{array}$ \\
\hline Age, $\mathrm{y} \pm \mathrm{SD}$ & $43.3 \pm 11.7$ & $39.2 \pm 13.4$ & $42.2 \pm 12.3$ & .049 \\
\hline Female gender, $\mathrm{n}(\%)$ & $58(49.2)$ & $24(51.1)$ & $82(49.7)$ & .86 \\
\hline Smokers, n (\%) & $12(10.2)$ & $15(31.9)$ & $27(16.4)$ & .001 \\
\hline $\begin{array}{l}\text { Positive TST } \\
\quad \text { (induration } \geq 10 \mathrm{~mm}), \\
\quad \mathrm{n}(\%)\end{array}$ & $4(3.4)$ & $29(61.7)$ & $33(20.0)$ & .001 \\
\hline \multicolumn{5}{|l|}{$\begin{array}{l}\text { No. of lymph nodes } \\
\text { sampled according } \\
\text { to stations }\end{array}$} \\
\hline $4 \mathrm{R}$ & 85 & 31 & 116 & \\
\hline $4 \mathrm{~L}$ & 8 & 3 & 11 & \\
\hline 7 & 110 & 34 & 144 & \\
\hline $10 \mathrm{R}$ & 8 & 5 & 13 & \\
\hline $10 \mathrm{~L}$ & 3 & 2 & 5 & \\
\hline $11 \mathrm{R}$ & 8 & 4 & 12 & \\
\hline $11 \mathrm{~L}$ & 47 & 9 & 56 & \\
\hline $3 \mathrm{P}$ & 1 & 0 & 1 & \\
\hline $\begin{array}{l}\text { Short-axis diameter } \\
\quad \text { on EBUS, } \mathrm{mm} \pm \mathrm{SD}\end{array}$ & $16.2 \pm 4.5$ & $16.5 \pm 4.5$ & $16.3 \pm 4.5$ & .64 \\
\hline $\begin{array}{r}\text { Short-axis diameter on } \\
\mathrm{CT} \text { chest, } \mathrm{mm} \pm \mathrm{SD}\end{array}$ & $20.2 \pm 7.1$ & $10.9 \pm 9.0$ & $20.4 \pm 7.6$ & .55 \\
\hline
\end{tabular}

$S D$, Standard deviation; $T S T$, tuberculin skin test; $E B U S$, endobronchial ultrasonography; $C T$, computed tomography.

\section{Lymph Node Data}

The overall size of the lymph nodes on EBUS ranged from 5 to $38 \mathrm{~mm}$ (short axis). There was no difference in the size of the lymph nodes at various stations measured on EBUS (or CT chest) in the 2 groups. A total of 358 lymph node stations (270 sarcoidosis, 88 tuberculosis) were examined in 165 patients. Among the endosonographic characteristics (Table 2), a heterogeneous echotexture of lymph nodes was significantly more common $(P<.001)$ in tuberculosis $(53.4 \%)$ than sarcoidosis $(12.6 \%)$. The coagulation necrosis sign was seen in $23(26.1 \%)$ lymph nodes with tuberculosis and $9(3.3 \%)$ of those with sarcoidosis, showing a statistically significant difference $(P<.001)$. The odds for diagnosis of tuberculosis were significantly higher in the presence of heterogeneous echotexture (odds ratio [OR], 7.96) or coagulation necrosis sign (OR, 10.26). The agreement for heterogeneous echotexture and coagulation necrosis between lymph nodes in the same patient ranged from 0.493 to 0.668 and from 0.381 to 0.658 , respectively ( $\kappa$ statistic).

\section{Differential Diagnosis}

Overall, the finding of heterogeneous echotexture was observed in $23(19.5 \%)$ and $28(59.6 \%)$ patients with sarcoidosis and tuberculosis, respectively; the coagulation necrosis sign was observed in $8(6.8 \%)$ and $16(34 \%)$ patients with sarcoidosis and tuberculosis, respectively (Table 3). The specificity of heterogeneous echotexture in any of the sampled lymph nodes for a diagnosis of tuberculosis was $80.5 \%$; the specificity of the coagulation necrosis sign was $93.2 \%$ (Table 3). A combination of a positive TST and either heterogeneous echotexture or coagulation necrosis sign had a specificity of $98 \%$ and a positive predictive value of $91 \%$ for a diagnosis of tuberculosis compared with sarcoidosis; the predictive values of the TST and sonographic characteristics depending on the prevalence of tuberculosis are depicted in Table 4. 
TABLE 2. Comparison of endosonographic characteristics of lymph nodes on endobronchial ultrasonography between sarcoidosis and tuberculosis

\begin{tabular}{|c|c|c|c|c|}
\hline & $\begin{array}{c}\text { Sarcoidosis } \\
\text { (270 lymph } \\
\text { nodes), } \\
\text { n }(\%) \\
\end{array}$ & $\begin{array}{c}\text { Tuberculosis } \\
\text { (88 lymph } \\
\text { nodes) } \\
\text { n }(\%) \\
\end{array}$ & $\begin{array}{l}\text { Odds ratio } \\
\quad(95 \% \\
\text { confidence } \\
\text { intervals }) \\
\end{array}$ & $\begin{array}{c}P \\
\text { value } \\
\end{array}$ \\
\hline \multicolumn{5}{|l|}{ Shape } \\
\hline Round & $38(14.1)$ & $17(19.3)$ & $1.46(0.78-2.75)$ & .24 \\
\hline Oval & $232(85.9)$ & $71(80.7)$ & & \\
\hline \multicolumn{5}{|l|}{ Margin } \\
\hline Distinct & $266(98.5)$ & $86(97.7)$ & $1.55(0.28-8.6)$ & .64 \\
\hline Indistinct & $4(1.5)$ & $2(2.3)$ & & \\
\hline \multicolumn{5}{|l|}{ Echogenicity } \\
\hline Homogeneous & $236(87.4)$ & $41(46.6)$ & $7.96(4.58-13.82)$ & $<.001$ \\
\hline Heterogeneous & 34 (12.6) & $47(53.4)$ & & \\
\hline \multicolumn{5}{|l|}{$\begin{array}{r}\text { Central hilar } \\
\text { structure }\end{array}$} \\
\hline Absent & $233(86.3)$ & 78 (88.6) & $0.81(0.38-1.70)$ & .72 \\
\hline Present & 37 (13.7) & $10(11.4)$ & & \\
\hline \multicolumn{5}{|l|}{$\begin{array}{l}\text { Coagulation } \\
\text { necrosis sign }\end{array}$} \\
\hline Absent & $261(96.7)$ & $65(73.9)$ & $10.26(4.53-23.23)$ & $<.001$ \\
\hline Present & $9(3.3)$ & $23(26.1)$ & & \\
\hline
\end{tabular}

On multivariate analysis, the presence of heterogeneous echotexture and a positive TST but not the coagulation necrosis sign were significant predictors of the diagnosis of tuberculosis (Table 5).

\section{Serious Adverse Events}

Two patients developed a pneumothorax after performing TBLB. One was managed with administration of high flow oxygen and the other underwent a single-time aspiration. All patients responded to the management and recovered. The EBUS TBNA procedure was shortened to only 3 passes in 1 patient because of excessive coughing. The aspirate in this patient revealed acid-fast bacilli on microscopic examination.

\section{DISCUSSION}

To the best of our knowledge, this is the first study to directly compare the EBUS characteristics of lymph nodes in patients with sarcoidosis and tuberculosis. In addition, it is the largest study to date to describe the endosonographic characteristics of lymph nodes in patients with either disease. The results of this study suggest that findings of heterogeneous echotexture and central necrosis in the lymph node on EBUS favor a diagnosis of tuberculosis over sarcoidosis. The positive predictive value for the diagnosis of tuberculosis is $91 \%$ when either of these findings is combined with a TST.

The recent advent of endoscopic ultrasonographic techniques (endoscopic ultrasonography and EBUS) has led to interest in the endosonographic characteristics of various diseases involving the mediastinal lymph nodes. The focus has primarily been on differentiating malignant from nonmalignant lymph nodes. ${ }^{11,18}$ Occasional reports have described findings for sarcoidosis and tuberculosis. ${ }^{19-22}$ This is the first study to directly compare the EBUS characteristics of lymph nodes in patients with sarcoidosis and tuberculosis. In a recent study by Imai and colleagues, ${ }^{19} 88 \%$ of the lymph nodes in sarcoidosis were homogeneous, similar to our study $(87.4 \%)$ and a majority had distinct margins. Most of the lymph nodes in our study were oval rather than round in contrast to the study by Imai and colleagues. ${ }^{19}$ A possible reason is that we classified the shape of the lymph node based on visual appearance, whereas Imai and colleagues ${ }^{19}$ used a long axis to short axis ratio of $2 \mathrm{~cm}$ or greater to define a node as oval. We further believe that measuring the axis of a lymph node parallel to the airway is difficult, because it frequently does not fit 1 field of ultrasonic view. The measurements thus may be erroneous and the visual impression of shape might be more reliable. We observed a lower proportion of sarcoidosis lymph nodes with a central hilar structure than previously reported for sarcoidosis or benign lymphadenopathy. ${ }^{11,19}$ This is because we considered a central hilar structure as a central hyperechoic structure without a central intranodal vessel.

We found a low sensitivity of the coagulation necrosis sign $(34 \%)$ similar to that reported by Rana and colleagues $^{20}(41 \%)$ for the diagnosis of tuberculosis. This was further reflected in the multivariate analysis where it

TABLE 3. Endobronchial ultrasonography node characteristics for the differential diagnosis of tuberculosis among patients with sarcoidosis and tuberculosis

\begin{tabular}{|c|c|c|c|c|}
\hline & Sensitivity, \% $(95 \%$ CI $)$ & Specificity, \% (95\% CI) & PPV, $\%(95 \%$ CI $)$ & NPV, \% $(95 \%$ CI $)$ \\
\hline Heterogeneous echotexture & $59.6(44.3-73.6)$ & $80.5(72.2-87.2)$ & $54.9(40.3-68.9)$ & $83.3(75.2-89.7)$ \\
\hline Coagulation necrosis sign & $34.0(20.9-49.3)$ & $93.2(87.1-97.0)$ & $66.7(44.7-84.4)$ & $78.0(70.3-84.6)$ \\
\hline $\begin{array}{l}\text { Heterogeneous echotexture or coagulation } \\
\text { necrosis sign }\end{array}$ & $61.7(46.4-75.5)$ & $78.8(70.3-85.8)$ & $53.7(39.6-67.4)$ & $83.8(75.6-90.1)$ \\
\hline Positive TST (induration $\geq 10 \mathrm{~mm}$ ) & $61.7(46.4-75.5)$ & $96.6(91.6-99.1)$ & $87.9(71.8-96.6)$ & $86.4(79.3-91.7)$ \\
\hline $\begin{array}{l}\text { Heterogeneous echotexture or coagulation } \\
\text { necrosis sign and positive TST }\end{array}$ & $42.6(28.3-57.8)$ & $98.3(94.01-99.8)$ & $90.9(70.8-98.9)$ & $81.1(73.7-87.2)$ \\
\hline
\end{tabular}

$C I$, Confidence interval; $N P V$, negative predictive value; $P P V$, positive predictive value; $T S T$, tuberculin skin test. 
TABLE 4. Predictive values of heterogeneous echotexture or coagulation necrosis sign on endobronchial ultrasonography and positive tuberculin skin test depending on the prevalence of tuberculosis

\begin{tabular}{lcc}
\hline Prevalence, \% & $\begin{array}{c}\text { Positive predictive } \\
\text { value, } \%(\mathbf{9 5} \% \mathbf{C I})\end{array}$ & $\begin{array}{c}\text { Negative predictive } \\
\text { value, \% }(\mathbf{9 5} \% \mathbf{C I})\end{array}$ \\
\hline 1 & $20.2(5.9-84.3)$ & $99.4(96.7-99.9)$ \\
5 & $56.9(15.6-91.7)$ & $97.0(93-99.1)$ \\
10 & $73.6(36-95.6)$ & $93.9(88.9-97.1)$ \\
20 & $86.2(60.1-98)$ & $87.3(80.8-92.2)$ \\
\hline
\end{tabular}

CI, Confidence interval.

was not found to be significant. This sonographic and radiological finding corresponds to the histologic presence of necrosis. However, the coagulation necrosis sign has a high specificity $(93.2 \%)$ for the diagnosis of tuberculosis. The presence of a heterogeneous lymph node, on the other hand, had a specificity of $80.5 \%$ for the diagnosis of tuberculosis. Agreement for heterogeneous echotexture and coagulation necrosis between lymph nodes in the same patient was poor suggesting that as many lymph nodes as possible should be sonographically evaluated to pick up these findings. Fujiwara and colleagues $^{11}$ and others ${ }^{16}$ have reported the coagulation necrosis sign as a predictor of malignant involvement, however in high tuberculosis burden areas, tuberculosis is an important differential for this sonographic feature. Although not evaluated in our study, the presence of a central hilar structure and/or central intranodal vessel may help in differentiating benign from malignant nodes in patients with the coagulation necrosis sign when malignancy is a consideration. ${ }^{11,18,23,24}$ Our study also reveals that the size, shape, margin, and presence or absence of a central hilar structure does not differentiate between tuberculosis and sarcoidosis. This is in contrast to the findings of Fritscher-Ravens and colleagues, ${ }^{21}$ who observed that tuberculous nodes are significantly smaller compared with sarcoidosis.

The differential diagnosis of tuberculosis and sarcoidosis remains a challenge especially in countries with a high tuberculosis burden. ${ }^{4}$ Serologic tests as well as qualitative nucleic acid amplification (NAA) tests for $M$ tuberculosis do not differentiate tuberculosis from sarcoidosis, ${ }^{25-27}$ unless quantitative NAA is performed. ${ }^{28}$ In this regard, the

TABLE 5. Multivariate logistic regression analysis on the predictors of the diagnosis of tuberculosis

\begin{tabular}{lcc}
\hline & $\begin{array}{c}\text { Adjusted odds ratio } \\
(\mathbf{9 5} \% \text { confidence intervals) }\end{array}$ & $\boldsymbol{P}$ value \\
\hline Heterogeneous echotexture & $3.04(1.06-8.68)$ & .038 \\
Coagulation necrosis sign & $1.93(0.49-7.58)$ & .349 \\
Positive TST (induration $\geq 10 \mathrm{~mm})$ & $33.16(10.05-109.38)$ & .0001 \\
\hline
\end{tabular}

TST, Tuberculin skin test.
TST is a good discriminator in differentiating tuberculosis from sarcoidosis; a positive TST is a strong indicator against a diagnosis of sarcoidosis. ${ }^{29,30}$ The results of this study suggest that sonographic findings of heterogeneous echotexture or coagulation necrosis sign along with a positive TST is a stronger discriminator than TST alone, favoring a diagnosis of tuberculosis (over sarcoidosis) with a specificity of more than $98 \%$.

Our study has certain limitations. This is a single-center study conducted in a region with a high prevalence of tuberculosis. Thus, the conclusions are applicable to similar settings only, where the differential diagnosis of the 2 conditions is the clinical issue. However, the endosonographic characteristics of either of the 2 diseases can be generalized. The determination of lymph node characteristics was performed in real time by operators in contrast to some studies in which the endosonographic images were saved for analysis of lymph node characteristics at a later date. ${ }^{11,18}$ Furthermore, the operators were not blinded to the clinical presentation and radiological picture of the patient. In addition, we did not follow any numeric criterion for defining shape and did not use any software for determination of echogenicity. This could be a source of potential subjectivity and bias in recording of the findings. However, this more closely mimics real-life clinical situations where operators perform the procedures without any specific criteria or software. In view of all the limitations, the model proposed by us needs to be validated in different centers.

In conclusion, sonographic features of lymph nodes on EBUS have poor sensitivity but reasonable specificity for differentiating tuberculosis and sarcoidosis. The finding of a heterogeneous lymph node echotexture or coagulation necrosis sign along with a positive TST favors a diagnosis of tuberculosis over sarcoidosis. This finding has an important clinical implication in areas with a high prevalence of tuberculosis, where a decision to start antituberculosis therapy can be made on the basis of EBUS findings and a positive TST.

\section{References}

1. Gupta D, Sharma OP. Sarcoidosis. In: Jindal SK, Shankar PS, Raoof S, Gupta D, Aggarwal AN, Agarwal R, eds. Textbook of Pulmonary \& Critical Care Medicine. 1st ed. New Delhi: Jaypee Brothers; 2010:1188-216.

2. Koyama T, Ueda H, Togashi K, Umeoka S, Kataoka M, Nagai S Radiologic manifestations of sarcoidosis in various organs. Radiographics. 2004;24:87-104.

3. Andreu J, Caceres J, Pallisa E, Martinez-Rodriguez M. Radiological manifestations of pulmonary tuberculosis. Eur J Radiol. 2004;51:139-49.

4. Gupta D, Agarwal R, Aggarwal AN, Jindal SK. Sarcoidosis and tuberculosis: the same disease with different manifestations or similar manifestations of different disorders. Curr Opin Pulm Med. 2012;18:506-16.

5. Khan A, Agarwal R, Aggarwal AN, Gupta N, Bal A, Singh N, et al, Blind transbronchial needle aspiration without an on-site cytopathologist: experience of 473 procedures. Natl Med J India. 2011;24:136-9.

6. Agarwal R, Aggarwal AN, Gupta D. Efficacy and safety of conventional transbronchial needle aspiration in sarcoidosis: a systematic review and meta-analysis. Respir Care. 2013;58:683-93. 
7. Trisolini R, Tinelli C, Cancellieri A, Paioli D, Alifano M, Boaron M, et al. Transbronchial needle aspiration in sarcoidosis: yield and predictors of a positive aspirate. J Thorac Cardiovasc Surg. 2008;135:837-42.

8. Yasufuku K, Chiyo M, Sekine Y, Chhajed PN, Shibuya K, Iizasa T, et al. Real-time endobronchial ultrasound-guided transbronchial needle aspiration of mediastinal and hilar lymph nodes. Chest. 2004;126:122-8.

9. Oki M, Saka H, Kitagawa C, Kogure Y, Murata N, Ichihara S, et al. Prospective study of endobronchial ultrasound-guided transbronchial needle aspiration of lymph nodes versus transbronchial lung biopsy of lung tissue for diagnosis of sarcoidosis. J Thorac Cardiovasc Surg. 2012;143:1324-9.

10. Agarwal R, Srinivasan A, Aggarwal AN, Gupta D. Efficacy and safety of convex probe EBUS-TBNA in sarcoidosis: a systematic review and meta-analysis. Respir Med. 2012;106:883-92.

11. Fujiwara T, Yasufuku K, Nakajima T, Chiyo M, Yoshida S, Suzuki M, et al. The utility of sonographic features during endobronchial ultrasound-guided transbronchial needle aspiration for lymph node staging in patients with lung cancer: a standard endobronchial ultrasound image classification system. Chest. 2010;138:641-7.

12. Memoli JS, El-Bayoumi E, Pastis NJ, Tanner NT, Gomez M, Huggins JT, et al. Using endobronchial ultrasound features to predict lymph node metastasis in patients with lung cancer. Chest. 2011;140:1550-6.

13. Curvo-Semedo L, Teixeira L, Caseiro-Alves F. Tuberculosis of the chest. Eur J Radiol. 2005;55:158-72.

14. Rusch VW, Asamura H, Watanabe H, Giroux DJ, Rami-Porta R, Goldstraw P. The IASLC lung cancer staging project: a proposal for a new international lymph node map in the forthcoming seventh edition of the TNM classification for lung cancer. J Thorac Oncol. 2009;4:568-77.

15. Srinivasan A, Agarwal R, Gupta N, Aggarwal AN, Gupta D. Initial experience with real time endobronchial ultrasound guided transbronchial needle aspiration from a tertiary care hospital in north India. Indian J Med Res. 2013;137:803-7.

16. Roberts SA, Mahon BS, Evans R. Coagulation necrosis in malignant mediastinal nodes on endoscopic ultrasound: a new endosonographic sign. Clin Radiol. 2005; 60:587-91.

17. Costabel U, Hunninghake GW. ATS/ERS/WASOG statement on sarcoidosis. Sarcoidosis Statement Committee. American Thoracic Society. European Respiratory Society. World Association for Sarcoidosis and Other Granulomatous Disorders. Eur Respir J. 1999;14:735-7.

18. Schmid-Bindert G, Jiang H, Kahler G, Saur J, Henzler T, Wang H, et al. Predicting malignancy in mediastinal lymph nodes by endobronchial ultrasound: a new ultrasound scoring system. Respirology. 2012;17:1190-8.
19. Imai $\mathrm{N}$, Imaizumi $\mathrm{K}$, Ando $\mathrm{M}$, Shimokata $\mathrm{T}$, Ogawa $\mathrm{T}$, Ito $\mathrm{S}$, et al. Echoic features of lymph nodes with sarcoidosis determined by endobronchial ultrasound. Intern Med. 2013;52:1473-8.

20. Rana SS, Bhasin DK, Srinivasan R, Singh K. Endoscopic ultrasound (EUS features of mediastinal tubercular lymphadenopathy. Hepatogastroenterology. 2011;58:819-23.

21. Fritscher-Ravens A, Ghanbari A, Topalidis T, Pelling M, Kon OM, Patel K, et al Granulomatous mediastinal adenopathy: can endoscopic ultrasound-guided fine-needle aspiration differentiate between tuberculosis and sarcoidosis? Endoscopy. 2011:43:955-61.

22. Lin SM, Chung FT, Huang CD, Liu WT, Kuo $\mathrm{CH}$, Wang $\mathrm{CH}$, et al Diagnostic value of endobronchial ultrasonography for pulmonary tuberculosis J Thorac Cardiovasc Surg. 2009;138:179-84.

23. Sawhney MS, Debold SM, Kratzke RA, Lederle FA, Nelson DB, Kelly RF Central intranodal blood vessel: a new EUS sign described in mediastinal lymph nodes. Gastrointest Endosc. 2007;65:602-8.

24. Satterwhite LG, Berkowitz DM, Parks CS, Bechara RI. Central intranoda vessels to predict cytology during endobronchial ultrasound transbronchial needle aspiration. J Bronchology Interv Pulmonol. 2011;18:322-8.

25. Mootha VK, Agarwal R, Ahmed J, Gupta D, Ahmed J, Verma I, et al. The Sarcoid-Tuberculosis link: evidence from a high TB prevalence country. J Infect. 2010;60:501-3.

26. Gupta D, Agarwal R, Aggarwal AN, Verma I. Immune responses to mycobacterial antigens in sarcoidosis: a systematic review. Indian J Chest Dis Allied Sci. 2011:53:41-9.

27. Barrios-Payan J, Saqui-Salces M, Jeyanathan M, Alcántara-Vazquez A Castañon-Arreola M, Rook G, et al. Extrapulmonary locations of mycobacterium tuberculosis DNA during latent infection. J Infect Dis. 2012 206:1194-205.

28. Zhou Y, Li HP, Li QH, Zheng H, Zhang RX, Chen G, et al. Differentiation of sarcoidosis from tuberculosis using real-time PCR assay for the detection and quantification of Mycobacterium tuberculosis. Sarcoidosis Vasc Diffuse Lung Dis. 2008;25:93-9.

29. Gupta D, Chetty M, Kumar N, Aggarwal AN, Jindal SK. Anergy to tuberculin in sarcoidosis is not influenced by high prevalence of tuberculin sensitivity in the population. Sarcoidosis Vasc Diffuse Lung Dis. 2003;20:40-5.

30. Smith-Rohrberg D, Sharma SK. Tuberculin skin test among pulmonary sarcoidosis patients with and without tuberculosis: its utility for the screening of the two conditions in tuberculosis-endemic regions. Sarcoidosis Vasc Diffuse Lung Dis. 2006;23:130-4. 